\title{
A Practical Tropospheric Scatter Model Using the Parabolic Equation
}

\author{
Herbert V. Hitney, Senior Member, IEEE
}

\begin{abstract}
A simple method is described that accounts for tropospheric scatter using the parabolic equation portion of the radio physical optics (RPO) model. RPO is a hybrid propagation model that combines ray-optics and parabolic-equation methods to assess realistic range-dependent tropospheric effects at frequencies from $100 \mathrm{MHz}$ to $20 \mathrm{GHz}$. A semiempirical scatter model adds a random refractive-index fluctuation to the mean refractive-index value at each height considered by the parabolic equation method. The results of this scatter model are compared with another scatter model and with a few sample radio measurements.
\end{abstract}

\section{INTRODUCTION}

$\mathbf{T}$ HE radio physical optics (RPO) model is a hybrid model to account for propagation effects over water that vary with range in the direction of propagation. Four independent spatial regions are considered in RPO. Low-elevation-angle and low-altitude portions of the solution use the parabolicequation (PE) method as described by Tappert [1] and Dockery [2]. For elevation angles above a small limiting angle, rayoptics (RO) methods are used to coherently construct the resultant field strength considering the direct and reflected paths through the specified atmosphere, taking full account of magnitude and phase changes caused by the refractiveindex profile. For angles above $5^{\circ}$ and very short ranges, a flat-earth model that ignores all refractive and earth-curvature effects is used. For the region below the RO limiting angle but above the PE solution, an extended-optics method has been developed that is initialized by the PE solution at the maximum PE height and propagates the solution to higher heights using RO methods. The boundaries between the four RPO regions are selected such that the solution is continuous from each region to the adjacent region. A more detailed description of RPO and comparisons with other models are given by Hitney [3]. This paper will concentrate on the PE region of RPO, even though some of the figures show results from the other regions.

In applying RPO, it became apparent that the PE model can predict propagation loss values, for example those caused by diffraction, that are far in excess of tropospheric scatter levels. Since it is the goal of RPO to give realistic predictions for as many applications as possible at all ranges and altitudes, it was decided that a troposcatter model should be added to RPO. Such a model will not generally affect radar applications, but can be very imporant to communication or intercept applications. One possibility considered was to add the semiempirical

Manuscript received April 30, 1992; revised February 5, 1993

The author is with the RDT\&E Divison, Code 543, Naval Command, Control and Ocean Surveillance Center, San Diego, CA 92152-7385.

IEEE Log Number 9211255 model used in the engineer's refractive effects prediction system (EREPS), described by Patterson et al. [4], which is based on a model by Yeh [5] but is adjusted by the frequencygain term described by Rice et al. [6]. The disadvantage of this approach is that it would add a considerable computational burden to RPO, since the troposcatter model must be evaluated at all ranges and altitudes in parallel with the PE model, in order to add the scatter results to the PE solution. Thus a more efficient method that would simulate scattering effects within the PE model was sought.

\section{SCATTER MODEL}

The usual approach to simulating scatter in the PE model is to use a phase screen as described by Martin and Flatté [7]. In this approach, each vertical field point is multiplied at each range step by a small random phase, which is directly related to a small random fluctuation in refractivity. After propagating through many such screens, the phase variations produce amplitude variations that simulate the scattering effects. Two recent applications of this method to tropospheric propagation are described by Levy and Craig [8] and Rouseff [9]. To realistically assess tropospheric scatter, the vertical random phase variations should have correlation properties in accordance with the Kolmogorov spectrum for vertical wave numbers between the inner and outer scales of turbulence in the inertial convective subrange. In the horizontal direction, however, the random fluctuations are usually assumed to be uncorrelated, since the PE range step is typically greater than the outer scale of turbulence. Ishimaru [10] gives the inner scale length as about $1 \mathrm{~mm}$ to $1 \mathrm{~cm}$ and the outer scale length as about 10 to $100 \mathrm{~m}$.

In the PE portion of the RPO model, field strength is computed at equally spaced vertical points separated by a height interval of $\delta z$. In RPO, $\delta z$ is a complicated function of frequency and the refractivity profile, and varies between approximately 0.5 and $18 \mathrm{~m} . \delta z$ is chosen for issues related to the numerical solution of both the RO and PE models, and is always greater than the inner scale length. Hence it would appear that a phase screen approach in RPO would be unworkable, since potentially important scattering from scale lengths less than $\delta z$ would be ignored. In spite of this consideration, a phase screen approach was implemented in which the vertical random refractivity fluctuations are derived from the refractive index spectrum at the turbulent wave number given by $k=2 \pi / \delta z$. For simplicity's sake, the random fluctuations are uncorrelated in the vertical direction but are constant, and hence perfectly correlated, in the horizontal direction. Both of 
these assumptions are exactly opposite to the usual approach. Hence, the effects of scatter are simulated in RPO as a random and uncorrelated series of tiny stratified layers superimposed on the mean refractivity field. Some discussion of why this seems to work will be given later in this paper.

The one-dimensional refractive-index spectrum in the inertial convective subrange is given by Gossard [11] as

$$
S_{n}(k)=0.249 C_{n}^{2} k^{-5 / 3},
$$

where $C_{n}^{2}$ is the refractive-index structure parameter (also referred to in the literature as the structure constant), which varies with altitude and air mass, and $k$ is in inverse meters. Gossard [12] has published typical height gradients of the refractive-index structure parameter in different air masses, based on radiosonde observations analyzed by Showalter [13]. Gossard [11] has summarized the existing observational data in his figure 3.6, taken from Doviak et al. [14], except for the addition of maritime conditions deduced from forward scatter data collected by Ames et al. [15]. Doviak et al. define an "effective" median structure parameter as

$$
C_{n e}^{2} \simeq 3.9 \times 10^{-15} e^{-z / 2000},
$$

where $z$ is the height above the surface in meters. The scatter model in RPO uses a random number from a uniform distribution with a mean of zero and normalized such that the variance of the difference between adjacent numbers is equal to $S_{n}(k)$. These random numbers represent the refractive-index fluctuation that is added to the mean refractive-index value at each vertical point in the PE model. Specifically, the random fluctuation in refractive index, $n_{f}$, added to the mean refractive index at each point is given by

$$
n_{f}=\frac{r-0.5}{0.408} \sqrt{S_{n}(k)},
$$

where $r$ is a uniformly distributed random number in the interval 0 to 1 , and $S_{n}(k)$ is evaluated from (1) and (2). The distribution of $n_{f}$ has the required mean of zero and variance described above.

Gossard's figure 3.6 [11] illustrates how various investigators have found or proposed a wide range of structure parameters versus altitude. The proper structure parameter profile to use in a particular case no doubt depends on air-mass considerations, the mean refractive-index profile, and possibly other factors. However, it is proposed that the effective median structure parameter profile specified by (2) will give reasonable results in the RPO model.

\section{RESULTS}

Figs. 1 and 2 compare RPO results with radio measurements reported by Ames et al. [15], presented as propagation loss versus range. Propagation loss is the ratio of transmitted to received power including antenna pattern effects but normalized to zero $\mathrm{dB}$ antenna gains. In this experiment, signal levels from a $220 \mathrm{MHz}$ source at $23.5 \mathrm{~m}$ above mean sea level were recorded versus range by an aircraft that flew an entirely overwater path from Scituate, Massachusetts, toward Sable Island. Figs. 1 and 2 illustrate data collected on 17 December 1953

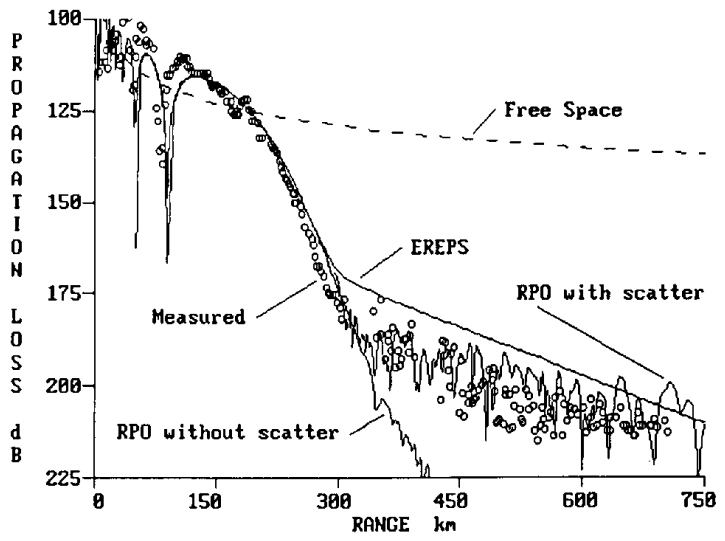

Fig. 1. Propagation loss versus range for $220 \mathrm{MHz}$, transmitter height $23.5 \mathrm{~m}$, and receiver height $3048 \mathrm{~m}$ at Scituate, MA, on 17 December 1953



Fig. 2. Propagation loss versus range for $220 \mathrm{MHz}$, transmitter height $23.5 \mathrm{~m}$, and receiver height $152 \mathrm{~m}$ at Scituate, MA, on 11 February 1954.

and 11 February 1954 with the aircraft at altitudes of 3048 $\mathrm{m}$ and $152 \mathrm{~m}$, respectively. RPO results with and without the scatter model are shown along with the EREPS model results compared with the measured data, which are indicated by small circles. Since measured refractivity profiles versus height were not available for these data, a standard atmosphere modified-refractivity gradient of $118 M$ units per kilometer was assumed in both RPO and EREPS. Note the modified refractivity $M=(n-1+z / a) \times 10^{6}$, where $n$ is refractive index, $z$ is height, and $a$ is the earth's radius. The RPO model without scatter significantly overestimates propagation loss at the longer ranges, while both the RPO model with scatter and the EREPS model match the measurements reasonably well.

Figs. 3, 4, and 5 show comparisons of the models with short-range, over-water measurements at 9653,3037 , and 1031 $\mathrm{MHz}$, respectively, collected on 23 March 1971, and indicated by small squares in the figures. This series of measurements was performed using horizontally polarized transmitters $3 \mathrm{~m}$ above sea level on a boat and receivers $4.7 \mathrm{~m}$ above sea level on the coast of Point Loma in the city of San Diego. The 


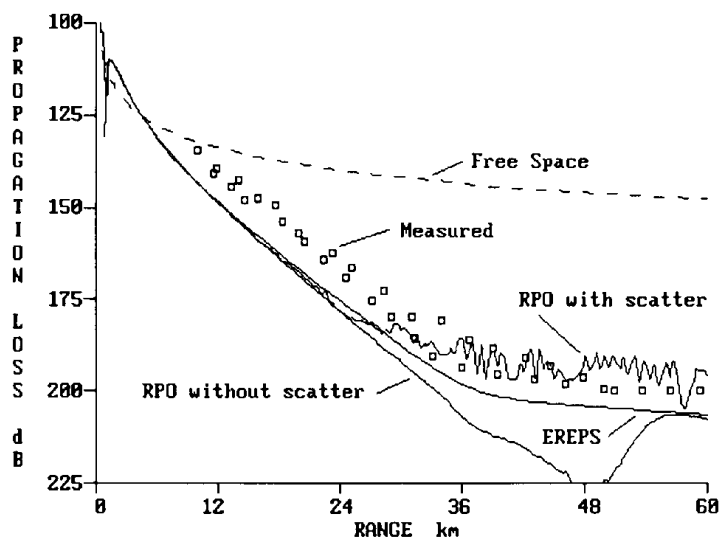

Fig. 3. Propagation loss versus range for $9653 \mathrm{MHz}$, transmitter height $3 \mathrm{~m}$, and receiver height $4.7 \mathrm{~m}$ at Point Loma on 23 March 1971.

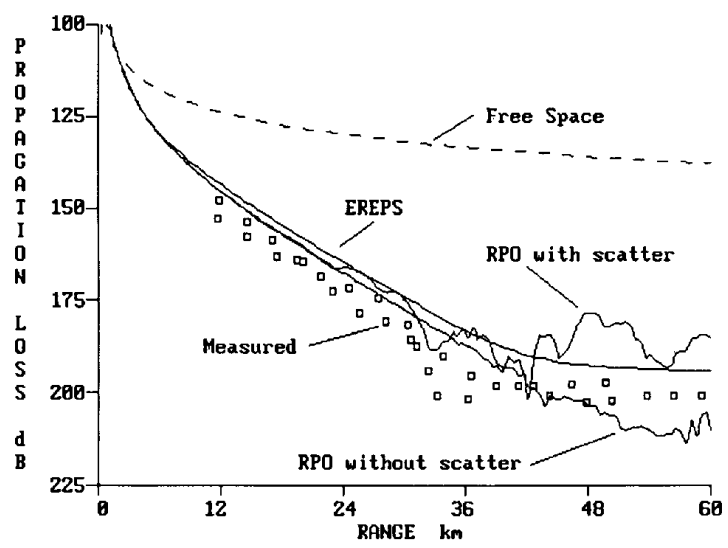

Fig. 4. Propagation loss versus range for $3037 \mathrm{MHz}$, transmitter height $3 \mathrm{~m}$, and receiver height $4.7 \mathrm{~m}$ at Point Loma on 23 March 1971.

refractive-index profile of the lower atmosphere was measured at the receiver site by radiosonde during the measurement period and is presented in Table I. This profile was very nearly standard, as defined above, except for a small trapping layer between 581 and $624 \mathrm{~m}$. The profile of Table I was used by RPO in preparation of the results shown in Figs. 3-5, while the EREPS model used the single standard gradient of $118 \mathrm{M}$ units per kilometer.

The RPO scatter model is at least as good as, and sometimes better than, the EREPS model in matching the measured data in Figs. 3-5. The match is good even for Figs. 3 and 4, which show small offsets from the models at all ranges, suggestive of unidentified errors of a few $\mathrm{dB}$ in the data. An interesting feature of the RPO results without scatter is the influence of the relatively high trapping layer, which appears as a decrease in propagation loss at the longer ranges at all frequencies. This effect is most pronounced at $1031 \mathrm{MHz}$, where there is not much difference between the RPO results with and without scatter. The effects of scatter in this case have clearly combined with the effects of the trapping layer to give a small



Fig. 5. Propagation loss versus range for $103 \mathrm{i} \mathrm{MHz}$, transmitter height $3 \mathrm{~m}$, and receiver height $4.7 \mathrm{~m}$ at Point Loma on 23 March 1971.

TABLE I

Modified Refractivity Versus Height Profile at PoINT Loma on 23 March 1971

\begin{tabular}{cc}
\hline Height $(\mathrm{m})$ & $M$ Units \\
\hline 0.0 & 317.7 \\
33.0 & 322.3 \\
291.8 & 358.5 \\
520.6 & 381.5 \\
580.8 & 387.4 \\
624.2 & 378.4 \\
838.2 & 394.0 \\
1328.8 & 455.4 \\
\hline
\end{tabular}

random fluctuation of the modeled loss around the values modeled without scatter.

Fig. 6 shows a comparison of the models with measurements at $3088 \mathrm{MHz}$ collected on 16 May 1974 . In this experiment, a horizontally polarized transmitter was placed $21 \mathrm{~m}$ above sea level on the coast of Point Loma, and the signal levels were received and recorded in an aircraft flying at $305 \mathrm{~m}$ altitude on a westward path entirely over water out to approximately 400 $\mathrm{km}$. The refractive-index profile was measured by radiosonde at the transmitter site on the day of the measurement and indicated that a trapping layer existed between 933 and 1006 $\mathrm{m}$. The measured profile is presented in Table II, and was used by RPO for the results plotted in Fig. 6, while the EREPS model assumed a standard atmosphere as before. The RPO model with and without scatter and the EREPS model all overestimate the measured loss, indicated by the dotted line. It is conjectured that scattering from stronger turbulence associated with the elevated duct and not taken into account by the median structure parameter assumed may be responsible for the higher signals in this case. It is also possible that the radiosonde underestimated the trapping layer strength in Table II, and the higher signals of Fig. 6 thus resulted from stronger ducting effects than modeled. Ducting effects can also be enhanced by refractive structure changes in range, which were not known for the case of Fig. 6. A recent paper by Hitney [16] examines these range-dependent effects for a case similar to the one presented in Fig. 6. 




Fig. 6. Propagation loss versus range for $3088 \mathrm{MHz}$, transmitter height $21 \mathrm{~m}$, and receiver height $305 \mathrm{~m}$ at Point Loma on 16 March 1974.

TABLE II

Modified Refractivity Versus Height Profile at Point Loma on 16 May 1974

\begin{tabular}{cc}
\hline Height $(\mathrm{m})$ & $M$ Units \\
\hline 0.0 & 321.4 \\
33.0 & 325.5 \\
681.7 & 406.2 \\
933.1 & 433.3 \\
1006.4 & 427.6 \\
1155.2 & 429.6 \\
1269.1 & 443.7 \\
1854.2 & 519.9 \\
\hline
\end{tabular}

\section{DISCUSSION}

The RPO scatter model appears to match the measurements examined at least as well as the EREPS model. Why this model works so well is somewhat of a mystery. It is believed that the approximate strength of the scatter is determined by (1), in spite of the uncorrelated fluctuations in the vertical, so long as $\delta z$ is between the inner and outer scale lengths of turbulence, which is almost certainly the case. The attenuation rate with range is determined by the structure parameter dependence on height given by (2), since greater ranges depend on scattering from greater heights. The perfect correlation of refractivity fluctuations in range is irrelevant for practical cases, since the mean refractivity profile is generally bending the ray paths to a different height level from one range step to the next; hence the net effect is uncorrelated fluctuations in the horizontal.

One objection to this model is that $\delta z$, which is selected based on RO and PE numerical solution issues, is used to fix the scale of turbulence that is considered. It would seem better to select $\delta z$ such that all important scales of turbulence would be considered in the solution. One justification of this model concerns the relationship between $\delta z$ and the maximum PE elevation angle, $\theta_{\max }$, which is given by

$$
\delta z=\frac{\lambda}{2} \frac{1}{\sin \left(\theta_{\max }\right)},
$$

where $\lambda$ is the radio wavelength. If one desires that the troposcatter levels be approximated best at the longest ranges possible in the model, then these levels will necessarily be associated with the maximum PE angle. Smaller angles will be associated with signals at shorter ranges, where troposcatter is less important than diffraction or ducting. According to the Bragg scatter condition, as stated by Gossard and Strauch [17], the scale of turbulence, $L$, is related to the scatter elevation angle $\theta$ (one-half the normal "scattering angle") by

$$
L=\frac{\lambda}{2} \frac{1}{\sin (\theta)}
$$

and therefore the scale of turbulence associated with the maximum PE angle is $\delta z$, based on (4). In addition, all scales less than $\delta z$ are associated with angles greater than $\theta_{\max }$, which will not contribute to the PE solution. Thus, once $\delta z$ is fixed for the PE model, it can be argued that $\delta z$ is also the dominant scale of turbulence at the greater ranges. Nonetheless, selecting a different $\delta z$ will result in a different troposcatter level for a given case. For example, changing $\delta z$ from the normal RPO value of $10 \mathrm{~m}$ to $5 \mathrm{~m}$ for the case of Fig. 2 reduces the troposcatter level an average of about $8 \mathrm{~dB}$, which in this case is still reasonably close to the measurements. The relative importance of this uncertainty in the model to uncertainties in the refractive-index profiles or in $C_{n e}^{2}$ for different air masses is not known.

Another concern with this troposcatter model is that the refractivity fluctuations may result in vertical refractivity gradients that violate assumptions implicit in the split-step PE method approximations. This appears not to be the case, since the maximum gradient from these fluctuations is less than $0.02 \mathrm{~N} / \mathrm{m}$, which is smaller in magnitude than the standard atmosphere gradient of $0.039 \mathrm{~N} / \mathrm{m}$. Note refractivity $N=$ $(n-1) \times 10^{6}$, where $n$ is the refractive index.

The effect of using a random number function to simulate refractivity fluctuations is apparent in the rapid fluctuations of the modeled propagation loss at longer ranges, which resemble the measurements better than the smooth decay of the EREPS model. This rapid fluctuation is probably beneficial in using RPO for operational assessments, since users will get a better feel for the variable nature of tropospheric scatter. On the other hand, it is clearly possible that users will confuse nulls from the single characterization of the scatter with nulls from features in the mean profile, such as ducting. One method to distinguish such features would be to ensemble average RPO results and indicate upper and lower bounds. The scatter effects would tend to average out, while the ducting effects would remain constant. Such a model, however, is considered too inefficient for RPO purposes.

The scatter model as implemented in RPO is very efficient. For most cases, the extra time required for the random fluctuation calculations is insignificant, since the random number function and the exponential function required for the height dependence of $C_{n e}^{2}$ are performed just once. A computational experiment was performed to see if there would be any substantial difference by applying new random fluctuations at each PE range step. Although the exact modeled loss values changed due to the slightly different random fluctuations, the average levels were unchanged. 
The choice of a uniform distribution over a perhaps more reasonable normal distribution was also made based on efficiency. Modeled results for several cases using a normal distribution showed that average loss levels were equal to those modeled using a uniform distribution.

\section{CONCLUSION}

The tropospheric scatter model described for RPO is a computationally efficient and simple alternative to the EREPS troposcatter model. This model should allow for realistic assessment of propagation effects for many applications and includes the ability to combine scattering with ducting or other nonstandard refractive effects.

\section{ACKNOWLEDGMENT}

The author would like to thank R. A. Paulus and K. D. Anderson for many helpful discussions while developing the model; E. E. Gossard for an initial review of this paper; and the Office of Naval Technology for its continuing support of radio propagation modeling efforts. The measurements presented in Figs. 3 through 6 were performed by the Naval Command, Control and Ocean Surveillance Center RDT\&E Division (then the Naval Electronics Laboratory Center).

\section{REFERENCES}

[1] F. D. Tappert, "The parabolic approximation method," in Wave Propa gation and Underwater Acoustics, J. B. Keller and J. S. Papadakis, Eds New York: Springer-Verlag, 1977.

[2] G. D. Dockery, "Modeling electromagnetic wave propagation in the troposphere using the parabolic equation," IEEE Trans. Antennas Propagat., vol. 36, no. 10 , pp. 1464-1470, 1988

[3] H. V. Hitney, "Hybrid ray optics and parabolic equation methods for radar propagation modeling," in Radar 92, IEE Conference Publication No. 365 , pp. 58-61, Brighton, UK, 12-13 Oct 92.

[4] W. L. Patterson et al., "Engineer's refractive effects prediction system (EREPS), Revision 2.0," Naval Ocean Syst. Center, San Diego, CA, Tech. Doc. 1342, Feb. 1990
[5] L. P. Yeh, "Simple methods for designing troposcatter circuits," IRE Trans. Comm. Syst., vol. CS-8, no. 3, pp. 193-198, 1960.

[6] P. L. Rice, A. G. Longley, K. A. Norton, and A. P. Barsis, "Transmission loss predictions for tropospheric communication circuits," National Bureau of Standards Tech. Note 101, May 7, 1965.

[7] J. M. Martin and S. M. Flatté, "Intensity images and statistics from numerical simulation of wave propagation in 3-D random media," Appl. Opt., vol. 27, no. 11, pp. 2111-2126, June 1, 1988.

[8] M. F. Levy and K. H. Craig, "Millimetre-wave propagation in the evaporation duct," AGARD CP-454, pp. 26.1-10, 1989.

[9] D. Rouseff, "Simulated microwave propagation through tropospheric turbulence," IEEE Trans. Antennas Propagat., vol. 40, no. 9, pp. $1076-1083,1992$.

[10] A. Ishimaru, Wave Propagation and Scattering in Random Media, vol. 2. New York: Academic Press, 1978, pp. 368-369.

[11] E. E. Gossard, "Radar research on the atmospheric boundary layer," in Radar in Meteorology, D. Atlas, Ed. Boston: American Meteorology Society, 1990.

[12] E. E. Gossard, "Refractive index variance and its distribution in different air masses," Radio Sci., vol. 12, no. 1, pp. 89-105, Jan-Feb 1977.

[13] A. K. Showalter, "Further studies of American air mass properties," Mon. Weather Rev., vol. 67, pp. 204-218, 1939.

[14] R. J. Doviak, R. M. Rabin, and A. J. Koscielny, "Doppler weather radar for profiling and mapping winds in the prestorm environment," IEEE Trans. Geosci, Remote Sens., vol. 21, pp. 25-33, 1983.

[15] L. A. Ames, P. Newman, and T. F. Rogers, "VHF tropospheric overwater measurements far beyond the horizon," PrOC. IRE, vol. 43, no. 10, pp. 1369-1373, 1955.

[16] H. V. Hitney, "Whispering gallery effects in the troposphere," Radio Sci., vol. 27, no. 6, pp. 893-898, Nov.-Dec. 1992.

[17] E. E. Gossard and R. G. Strauch, Radar Observation of Clear Air and Clouds. New York: Elsevier, 1983, p. 25.

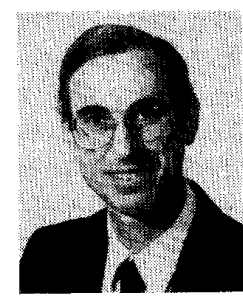

Herbert V. Hitney (SM'91) received the B.A degree in mathematics from San Diego State University, San Diego, CA, in 1966.

$\mathrm{He}$ has worked in the Research Development, Test and Evaluation Division of the Naval Command, Control and Ocean Surveillance Center (and predecessor organizations) since 1967 on a variety of theoretical and experimetal tropospheric radio propagation projects.

Mr. Hitney is a Life Member of the American Society of Naval Engineers and a member of Commission $\mathrm{F}$ of the U.S. National Committee of the International Union of Radio Science (USNC/URSI). 